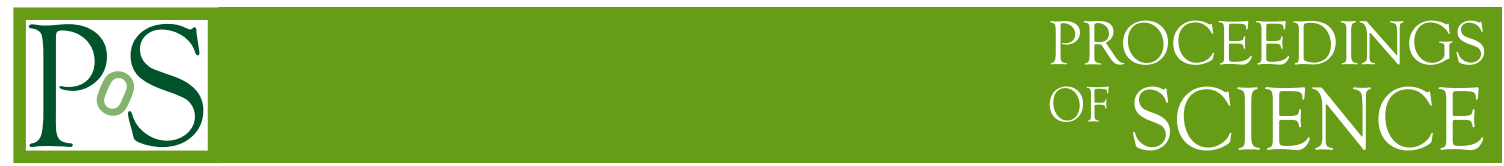

\title{
Unitarity Methods For Massless One-Loop Amplitudes
}

\author{
Warren B. Perkins* \\ Swansea University UK \\ E-mail: w.perkins@swan.ac.uk
}

\section{Edmund Warrick}

Swansea University $U K$

E-mail: 447153 @swan.ac.uk

Unitarity methods provide an efficient approach for calculating one-loop QCD amplitudes. Recent developments of these techniques have yielded a range of systematic implementations of the method. In particular the canonical basis approach is discussed and illustrated using seven gluon scattering.

XII Advanced Computing and Analysis Techniques in Physics Research November 3-7, 2008

Erice, Italy

\footnotetext{
${ }^{*}$ Speaker.
} 


\section{Introduction}

New physics signals at the LHC must be extracted from large QCD backgrounds that must be known beyond leading order. While Feynman diagram techniques provide an in principle method of performing these NLO calculations, these techniques become increasingly unwieldy as the number of external particles increases due to a dramatic growth in the number of diagrams that must be calculated. Unitarity methods provide an efficient alternative for calculating amplitudes with large numbers of external particles. In this article we briefly review the underlying technique before discussing recent implementations. In particular we present the canonical basis approach [1] which yields compact, manifestly rational expressions for one-loop amplitudes. We illustrate this approach with reference to seven gluon scattering.

\section{The Structure of One-Loop Amplitudes}

Although we don't actually use Feynman diagram techniques in our calculations, the fact that these techniques exist does provide information about the form of one-loop amplitudes which we can use to specify the structure of the amplitudes we are interested in. Several of the steps outlined in this section are applicable to both the Unitarity and Feynman diagram approaches.

\section{Colour Ordering}

The full tree-level amplitudes for $n$ external gluons, $\mathscr{A}_{n}^{\text {tree }}$, in a $U\left(N_{c}\right)$ or $S U\left(N_{c}\right)$ gauge theory can be obtained by summing over a set of colour-ordered partial amplitudes, $A_{n}^{\text {tree }}(\sigma)$, multiplied by an associated colour-trace (for a review see [2]),

$$
\mathscr{A}_{n}^{\text {tree }}\left(\left\{k_{i}, \lambda_{i}, a_{i}\right\}\right)=g^{n-2} \sum_{\sigma \in S_{n} / Z_{n}} \operatorname{Tr}\left(T^{a_{\sigma(1)}} \cdots T^{a_{\sigma(n)}}\right) \times A_{n}^{\text {tree }}\left(k_{\sigma(1)}^{\lambda_{\sigma(1)}}, \ldots, k_{\sigma(n)}^{\lambda_{\sigma(n)}}\right)
$$

where $k_{i}, \lambda_{i}$, and $a_{i}$ are respectively the momentum, helicity $( \pm)$ and colour-index of the $i$-th external gluon and $g$ is the coupling constant. $S_{n} / Z_{n}$ is the set of non-cyclic permutations of $\{1, \ldots, n\}$ and the $U\left(N_{c}\right)\left(S U\left(N_{c}\right)\right)$ generators, $T^{a}$, are normalized such that $\operatorname{Tr}\left(T^{a} T^{b}\right)=\delta^{a b}$.

A similar decomposition is possible at one-loop [3]. In this case there are two traces over colour matrices and one must also sum over the different spins, $J$, of the internal particles circulating in the loop.

All of the contributions to a one-loop amplitude can be obtained by summing permutations of the leading in colour amplitudes [4], hence we focus on these.

\section{Integral Reduction}

In the Feynman diagram language, each contribution to the one-loop amplitude is of the form,

$$
I_{n}\left(f^{p}(k)\right)=\int d^{4} k \frac{f^{p}(k)}{\prod_{i=1}^{n}\left(\left(k-P_{i}\right)^{2}+i \varepsilon\right)},
$$

where $f^{p}(k)$ is a polynomial of degree $p$ in the loop momentum, $k$. Using propagator factors as a basis for terms in the numerator allows a simultaneous reduction in the degree of the numerator polynomial and the number of propagators,

$$
I_{n}\left(f^{p}(k)\right) \rightarrow \sum_{j} C_{j} I_{n-1}^{j}\left(f^{p-1}(k)\right) .
$$


In general this process can be iterated to yield a sum of pentagon integrals [5]. Further reduction is possible at the integral level [5, 6]. For massless theories reduction allows each amplitude to be expressed in the form,

$$
A_{n}^{1-\text { loop }}=\sum_{i} c_{i} I_{4}^{i}(1)+\sum_{j} d_{j} I_{3}^{j}(1)+\sum_{k} e_{k} I_{2}^{k}(1)+R,
$$

where $I_{4}^{i}(1), I_{3}^{j}(1)$ and $I_{2}^{k}(1)$ are scalar box, triangle and bubble functions respectively and $c_{i}, d_{j}, e_{k}$ and $R$ are rational functions of spinor products.

\section{Supersymmetric Decomposition}

In super-Yang-Mills theories the contributions to gluon scattering due to different supermultiplets circulating in the loop can be decomposed into single particle contributions,

$A_{n}^{\mathcal{N}=1 \text { vector }} \equiv A_{n}^{[1]}+A_{n}^{[1 / 2]}, \quad A_{n}^{\mathcal{N}=1 \text { chiral }} \equiv A_{n}^{[1 / 2]}+A_{n}^{[0]}, \quad A_{n}^{\mathscr{N}=4} \equiv \mathrm{A}_{n}^{[1]}+4 A_{n}^{[1 / 2]}+3 A_{n}^{[0]}$.

For spin-0 we always consider a complex scalar. Throughout we assume the use of a supersymmetry preserving regulator. Inverting these relations we have,

$$
A_{n}^{[1]}=A_{n}^{\mathscr{N}=4}-4 A_{n}^{\mathscr{N}=1 \text { chiral }}+A_{n}^{[0]},
$$

which expresses the amplitude of interest in terms of two supersymmetric amplitudes and one with a complex scalar circulating in the loop [4].

For supersymmetric amplitudes (1.4) simplifies due to cancellations within the loop-momentum integrals. For $\mathscr{N}=1$ amplitudes the purely rational term, $R$, is absent [7], while for $\mathscr{N}=4$ amplitudes only boxes appear [4].

\section{Spinor Helicity}

For a massless momentum, $k^{i}$, we can define two component commuting spinor variables via,

$$
\lambda_{m}^{i} \bar{\lambda}_{\dot{n}}^{i}=k_{v}^{i} \sigma_{m \dot{n}}^{v}
$$

For massless Weyl spinors $\left|i^{ \pm}\right\rangle$with momentum $k_{i}$ and chirality \pm we can define spinor innerproducts $[8,2]$,

$$
\langle i j\rangle \equiv\left\langle i^{-} \mid j^{+}\right\rangle=\varepsilon^{m n} \lambda_{m}^{i} \lambda_{n}^{j}, \quad[i j] \equiv\left\langle i^{+} \mid j^{-}\right\rangle=-\varepsilon^{i \dot{m}} \tilde{\lambda}_{\dot{m}}^{i} \tilde{\lambda}_{\dot{n}}^{j} .
$$

These spinor products are related to the momentum invariants by $\langle i j\rangle[j i]=2 k_{i} \cdot k_{j} \equiv s_{i j}$.

Colour-ordered amplitudes for tree level gluon scattering are particularly simple in this language: amplitudes with less than two distinct helicity legs vanish and the maximally helicity violating (MHV) amplitudes are given by the Parke-Taylor formula [9],

$$
A_{n}^{\text {tree }}\left(1^{ \pm}, 2^{+}, \ldots, n^{+}\right)=0, \quad A_{n}^{\text {tree }}\left(1^{+}, . ., j^{-}, . ., k^{-}, . ., n^{+}\right)=i \frac{\langle j k\rangle^{4}}{\langle 12\rangle\langle 23\rangle \cdots\langle n 1\rangle}
$$

\section{Unitarity Methods}

The Unitarity method utilises the process of cutting propagators [10]. To cut a propagator $\left((l-Q)^{2}+i \varepsilon\right)^{-1}$ we insert a factor of $\left((l-Q)^{2}+i \varepsilon\right) \delta\left((l-Q)^{2}\right)$ into the loop momentum integral 
and thus focus on the behaviour of the loop integral in the vicinity of the pole in this propagator. A pair of propagators, $\left((l-Q)^{2}+i \varepsilon\right)^{-1}$ and $\left((l-Q-P)^{2}+i \varepsilon\right)^{-1}$, in the integrand of the full loop integral will introduce (poly-)logarithms involving $P^{2}$ into the full integral. Cutting these propagators reproduces the coefficients of these logarithms [7]. The coefficients $c_{i}, d_{j}$ and $e_{k}$ in (1.4) can thus be determined by analysing all possible cuts of two propagators. The full amplitude, including the rational pieces, can be computed if the cut legs are kept in $4-2 \varepsilon$ dimensions [11] or by using on-shell recursion [12].

Cutting two propagators in a Feynman diagram splits the integrand into a product of two onshell factors. Summing all the Feynman diagrams gives tree amplitudes on either side of the cut (Fig.1) and the cut integral takes the form,

$$
C=\int D L I P S A_{\text {left }}^{\text {tree }}\left(-l_{1}, 1,2, . ., m, l_{2}\right) \times A_{\text {right }}^{\text {tree }}\left(-l_{2}, p, . ., s, l_{1}\right) .
$$

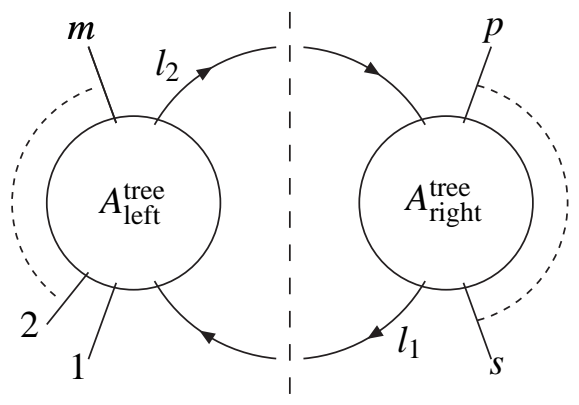

Fig.1 A double cut forms two tree amplitudes

Evaluating/manipulating the cut integral allows us to identify the coefficients of those boxes, triangles and bubbles on the right had side of (1.4) which contain these two propagators.

Cutting two propagators restricts the loop momentum to the surface of either a sphere or hyperboloid. Cutting more propagators further restricts the cut integration and the number of basis functions involved. Cutting three propagators leaves a one dimensional integral which yields some box and a specific triangle coefficient [13], while cutting four propagators replaces the integration with a sum over one or two momenta and algebraically yields a specific box coefficient [14].

In recent years considerable advances have been made in systematising the process of extracting the coefficients of the basis integral functions. Analytic implementations have used fermionic integration [15], direct extraction [16] and integrand level reduction [17]. The last of these has been implemented numerically [18] as has a variant that employs integer-dimension cuts to determine complete amplitudes, i.e. including the rational pieces [19]. A further numerical implementation uses on-shell recursion to compute complete amplitudes [20].

\section{The Canonical Basis Approach}

The approach we adopt recognises that there are a limited number of distinct structures that appear in the cut integral. By determining the contribution of each structure to the relevant coefficients we construct a canonical basis that can be used for any amplitude. This approach produces compact, explicitly rational results. We use a top down strategy, using quadruple cuts to determine the box coefficients, triple cuts for the triangles and traditional double cuts for the bubbles. We illustrate the process with some example bubble calculations. 


\section{Simple Canonical Forms}

Consider the following term that can arise in the cut integrand:

$$
\mathscr{H}_{1}=\frac{\langle a l\rangle}{\langle b l\rangle}=\frac{[b|l| a\rangle}{\left(l+k_{b}\right)^{2}} .
$$

This is a linear triangle contribution which reduces to a scalar triangle plus a bubble contribution. As discussed above, we choose to obtain the triangle contribution from a triple cut and extract just the bubble contribution from (1.11). The particular method used to determine a given canonical form is not important, explicit integration is straight forward in this case.

$$
\left.\mathscr{H}_{1}\right|_{\text {bubble }} \equiv H_{1}=\frac{[b|P| a\rangle}{[b|l| b\rangle} .
$$

The normalisation is such that an integrand of 1 contributes 1 to the bubble coefficient. Similarly,

$$
\mathscr{G}_{1}=\frac{[B l]\langle l c\rangle\langle l d\rangle}{\langle l a\rangle(l+Q)^{2}}
$$

gives box, triangle and bubble contributions.

$$
\left.\mathscr{G}_{1}\right|_{\text {bubble }} \equiv G_{1}=\left(\frac{[B|P[Q, P]| a\rangle\langle c|[Q, P]| d\rangle}{8 \Delta\langle a|Q P| a\rangle}-\frac{[B|P| a\rangle(\langle c a\rangle[a|P| d\rangle+\langle d a\rangle[a|P| c\rangle}{2\langle a|Q P| a\rangle[a|P| a\rangle}\right),
$$

where, $\Delta=P \cdot Q^{2}-P^{2} Q^{2}$.

The full basis requires forms with higher powers of loop momenta in the numerator. These can be found at http://pyweb.swan.ac.uk/ dunbar/sevengluon.html.

\section{A real calculation}

As an example of a real calculation we take the amplitude $A^{\mathscr{N}=1}\left(1^{-}, 2^{+}, 3^{-}, 4^{+}, 5^{+}, 6^{+}, 7^{-}\right)$ and consider the $(1,2)$ cut. To evaluate this cut we need two trees: one is 4-pt MHV and the other is 7-pt NMHV. The latter is naturally expressed as a sum of six terms. We will analyse one of these terms explicitly to illustrate the technique:

$$
A_{\text {piece }}^{\text {tree }}\left(l_{1}^{-H},-l_{2}^{H}, 3^{-}, 4^{+}, 5^{+}, 6^{+}, 7^{-}\right)=i \frac{\left[4\left|P_{56}\right| 7\right\rangle^{3}}{\langle 56\rangle\langle 67\rangle[34]} \frac{\left[4\left|P_{56}\right| 7\right\rangle^{3}\left\langle 7 l_{2}\right\rangle^{2-H}\left\langle 7 l_{1}\right\rangle^{1+H}}{\left\langle l_{1} l_{2}\right\rangle\left[3\left|\left(l_{1}-l_{2}\right)\right| 7\right\rangle\left\langle 7\left|P_{56} P_{34}\right| l_{2}\right\rangle\left\langle 7\left|\left(l_{1}-l_{2}\right) P_{34}\right| 5\right\rangle} .
$$

The corresponding cut integrand is then,

$$
\mathscr{C}=\frac{\left[4\left|P_{56}\right| 7\right\rangle^{3}}{s_{12}[12]\langle 56\rangle\langle 67\rangle[34]\left[3\left|P_{12}\right| 7\right\rangle\left\langle 7\left|P_{12} P_{34}\right| 5\right\rangle} \sum_{H} \frac{\left[2 l_{1}\right]^{2+H}\left[2 l_{2}\right]^{1-H}\left\langle 7 l_{2}\right\rangle^{2-H}\left\langle 7 l_{1}\right\rangle^{1+H}}{\left[l_{1} 1\right]\left\langle 7\left|P_{56} P_{34}\right| l_{2}\right\rangle} .
$$

As we would expect for a supersymmetric theory, summing over the spins of the internal particles reduces the degree of the numerator polynomial,

$$
\mathscr{C} \rightarrow \frac{\left[4\left|P_{56}\right| 7\right\rangle^{3}\langle 17\rangle^{2}}{\langle 21\rangle\langle 56\rangle\langle 67\rangle[34]\left[3\left|P_{12}\right| 7\right\rangle\left\langle 7\left|P_{12} P_{34}\right| 5\right\rangle} \frac{\left\langle 1 l_{2}\right\rangle\left\langle 7 l_{2}\right\rangle}{\left\langle 2 l_{2}\right\rangle\left\langle 7\left|P_{56} P_{34}\right| l_{2}\right\rangle} .
$$


Partial fractioning gives two terms of the $H_{1}$ type which are readily evaluated using (1.12), $C=\frac{\left[4\left|P_{56}\right| 7\right\rangle^{3}\langle 17\rangle^{2}}{\langle 21\rangle\langle 56\rangle\langle 67\rangle[34]\left[3\left|P_{12}\right| 7\right\rangle\left\langle 7\left|P_{12} P_{34}\right| 5\right\rangle} \times \frac{1}{\left\langle 7\left|P_{56} P_{34}\right| 2\right\rangle}\left(\langle 17\rangle-\frac{\left\langle 1\left|P_{34} P_{56}\right| 7\right\rangle\left[7\left|P_{56} P_{34} P_{12}\right| 7\right\rangle}{\left[7\left|P_{56} P_{34} P_{12} P_{34} P_{56}\right| 7\right\rangle}\right)$. (1.18)

The contributions from the other terms in this and the other $\mathscr{N}=17$-pt amplitudes can be calculated in a similar fashion and involve only the $H_{1}$ and $G_{1}$ forms. These results are available at http://pyweb.swan.ac.uk/ dunbar/sevengluon.html.

\section{Acknowledgements}

The authors would like to thank Dave Dunbar for many fruitful discussions. This work was supported by the STFC of the UK.

\section{References}

[1] D. C. Dunbar, W. B. Perkins and E. Warrick, arXiv:0903.1751 [hep-ph].

[2] M. Mangano and S.J. Parke, Phys. Rep. 200301 (1991).

[3] Z. Bern and D.A. Kosower, Nucl. Phys. B 362, 1991 (389).

[4] Z. Bern, L. J. Dixon, D. C. Dunbar and D. A. Kosower, Nucl. Phys. B 425 (1994) 217 [arXiv:hep-ph/9403226].

[5] W. L. van Neerven and J. A. M. Vermaseren, Phys. Lett. B 137 (1984) 241.

[6] Z. Bern, L. J. Dixon and D. A. Kosower, Phys. Lett. B 302 (1993) 299 [Erratum-ibid. B 318 (1993) 649] [arXiv:hep-ph/9212308],

G. Passarino and M. J. G. Veltman, Nucl. Phys. B 160 (1979) 151.

[7] Z. Bern, L. J. Dixon, D. C. Dunbar and D. A. Kosower, Nucl. Phys. B 435 (1995) 59 [arXiv:hep-ph/9409265].

[8] Z. Xu, D.-H. Zhang and L. Chang, Nucl. Phys. B 291, 1987 (392).

[9] S.J. Parke and T.R. Taylor, Phys. Rev. Lett. 56:1986 (2459).

[10] R. E. Cutkosky, J. Math. Phys. 1 (1960) 429.

[11] W. L. van Neerven, Nucl. Phys. B 268 (1986) 453.

[12] Z. Bern, L. J. Dixon and D. A. Kosower, Phys. Rev. D 73 (2006) 065013 [arXiv:hep-ph/0507005].

[13] Z. Bern, L. J. Dixon and D. A. Kosower, Nucl. Phys. B 513 (1998) 3 [arXiv:hep-ph/9708239], S. J. Bidder, N. E. J. Bjerrum-Bohr, D. C. Dunbar and W. B. Perkins, Phys. Lett. B 612 (2005) 75 [arXiv:hep-th/0502028],

N. E. J. Bjerrum-Bohr, D. C. Dunbar and W. B. Perkins, JHEP 0804 (2008) 038 [arXiv:0709.2086 [hep-ph]].

[14] R. Britto, F. Cachazo and B. Feng, Nucl. Phys. B 725 (2005) 275 [arXiv:hep-th/0412103].

[15] R. Britto, B. Feng and G. Yang, arXiv:0803.3147 [hep-ph] (and references therein)

[16] D. Forde, Phys. Rev. D 75, 125019 (2007) [arXiv:0704.1835 [hep-ph]].

[17] G. Ossola, C. G. Papadopoulos and R. Pittau, Nucl. Phys. B 763 (2007) 147 [arXiv:hep-ph/0609007].

[18] G. Ossola, C. G. Papadopoulos and R. Pittau, JHEP 0803 (2008) 042 [arXiv:0711.3596 [hep-ph]].

[19] R. K. Ellis, W. T. Giele, Z. Kunszt, K. Melnikov and G. Zanderighi, arXiv:0810.2762 [hep-ph].

[20] C. F. Berger et al., Phys. Rev. D 78, 036003 (2008) [arXiv:0803.4180 [hep-ph]]. 